\title{
LE FÉMININ ET LA DIFFÉRENCE DES SEXES
}

\author{
Rajaa Stitou*
}

\section{Résumé}

L'être féminin ou masculin n'est pas seulement une affaire d'anatomie ou de biologie. Il est lié à la subjectivité, mais il porte aussi la marque d'une culture. Les rites et les mythes collectifs dont la fonction est de donner du sens à l'énigme du féminin et à ce qui différencie les sexes en témoignent avec force. Or que deviennent ces rites, dans lesquels chaque sujet s'implique singulièrement, face aux mutations culturelles? La pratique clinique auprès de sujets immigrés, confrontés à un monde nouveau, qui n'abrite plus les mêmes rites et les mêmes ancrages symboliques, est riche en enseignements. Je chercherai à démontrer, en prenant appui sur mon expérience orientée par la psychanalyse et basée sur l'écoute du sujet dans son articulation au lien social, que ces transformations de repères ne sont pas sans répercussions subjectives, surtout lorsque le sujet ne se réfere pas à d'autres repères ou modèles identificatoires lui permettant de se reconnaître dans sa condition d'être sexué et d'accepter sa différence.

Mots-clés: féminin; différence des sexes; subjectivité; mutations culturelles.

\section{Resumo}

Ser homem ou mulher não é apenas uma questão de anatomia ou biologia. Ela está relacionada com a subjetividade, mas também traz a marca de uma cultura. Testemunha disso são os rituais e mitos coletivos, cuja função é dar sentido ao mistério das mulheres e ao que diferencia os sexos. Mas o que acontece a estes ritos, nos quais cada sujeito está envolvido particularmente, face às mudanças culturais? A prática clínica com sujeitos imigrantes, confrontados a um mundo novo que já não oferece os mesmos ritos e as mesmas âncoras simbólicas, é instrutiva. Tentaremos

* Psychanalyste, Maître de conférences en psychopathologie Université Montpellier III, Université d'Aix-Marseille 1. Email: rstitou@wanadoo.fr. 
mostrar, com base na experiência orientada pela psicanálise e baseada na escuta do sujeito em sua articulação com o laço social, que essas transformaçóes trazem repercussóes subjetivas, sobretudo quando o sujeito não se refere a outras referências ou modelos identificatórios que lhe permitam se reconhecer em sua condição de ser sexuado e aceitar suas diferenças.

Palavras-chave: sexo feminino; diferença de gênero; subjetividade; mudança cultural.

Abstract

FEMININITY AND THE DIFFERENCE BETWEEN THE SEXES

Femininity and the difference between the sexes are examined in their relationship to subjectivity and to cultural constructions, the forms of which change depending on the time and place. These constructions, which each subject must appropriate in a singular way and according to his or her own history, represent a certain necessity. They allow us to give meaning to the enigma of sex. However, they can also expose us to the risks of a subjective collapse when they become detached from their symbolic support. I will try to show, relying on my experience oriented by psychoanalysis and based on listening the subject in his articulation of the social link that these transformations are not without subjective consequences, especially when the subject does not refer other benchmarks or role models that allow to recognize his status as a sexual being and to accept their differences.

Keywords: femininity; difference between the sexes; subjectivity; cultural change.

\section{Resumen}

LO FEMENINO Y LAS DIFERENCIAS DE GÉNERO

Ser hombre o mujer no es sólo una cuestión de anatomía o biología. Está relacionado a la subjetividad, pero también lleva la marca de una cultura. Los rituales y mitos colectivos cuya función es dar sentido al misterio de la mujer y a lo que diferencia los sexos lo han demostrado vívidamente. Sin embargo iqué les ocurre a estos ritos, en los que cada sujeto está implicado singularmente, frente a los cambios culturales? La práctica clínica ejercida con sujetos inmigrantes, que se enfrentan a un mundo nuevo, un mundo que ya no les ofrece los mismos ritos ni los mismos anclajes simbólicos, es instructivo. Voy a tratar de demostrar, a partir de mi experiencia orientada por el psicoanálisis y basada en la escucha del sujeto en su articulación con el vinculo social, que esas transformaciones de anclajes tiene

Psic. Clin., Rio de Janeiro, vol. 25, N.I, P. I 97 - 2 I I, 2013 
repercusiones subjetivas, especialmente cuando el sujeto no se refiere a otros anclajes o modelos de identificación que le permitirían reconocerse en su condición de ser sexuado y aceptar su diferencia. culturales.

Palabras clave: femenino; diferencias de género; subjetividad; cambios

\section{Introduction}

En inscrivant la sexualité au cœur de la vie psychique, Freud l'a référée à une faille subjective qui concerne chaque sujet quant à son rapport au désir et à l'inconscient. Cette faille, c'est ce qui fait qu'aucune réponse, aucun savoir assuré ne peut épuiser la question: qu'est-ce qu'un homme? Qu'est-ce qu'une femme? La réponse à cette question ne peut se faire qu'à travers le langage, mais le langage rate à tout dire. Les mots eux-mêmes contiennent du manque et ne peuvent correspondre totalement aux choses qu'ils désignent. D'un point de vue psychanalytique, l'être féminin ou masculin n'est donc pas seulement une affaire d'anatomie ou de biologie. Cela relève d'une assomption subjective qui prend appui sur la culture, en passant par le langage mais sans y être réductible. En effet, toutes les cultures donnent un statut au féminin et au masculin. Ces constructions socioculturelles sont nécessaires, mais elles ne peuvent se comprendre sans la prise en compte de cette autre dimension évoquée plus haut, à savoir cette faille, cette incomplétude. Celle-ci est à la base de toute subjectivité, et fait qu'en rencontrant son désir, le sujet parlant rencontre son manque, en lui-même d'abord et dans son rapport à l'autre ${ }^{1}$. La différence des sexes, qui renvoie à la différence tout court et sans laquelle aucun lien social n'est possible, ne peut être appréhendable qu'à partir de ce manque.

Si la "libido est d'essence masculine", comme l'énonce Freud (1923/1969, p. 114), nous pouvons d'emblée souligner que l'énigme, chez l'homme ou la femme, est toujours féminine. Ce qui distingue l'un et l'autre sexe, c'est la manière de se positionner par rapport à cette énigme qui constitue la part rebelle, insoumise aux lois du langage, et qui touche chacun dans son mode de jouissance. C'est pourquoi Lacan affirme que "pas tout du féminin est inscrit dans la fonction phallique" (1972-73/1975, p.67). Selon cet auteur, le "phallus", qui est avant tout à entendre dans sa dimension signifiante, c'est-à-dire en référence aux lois de l'énonciation et du langage, n'a pas d'équivalent dans l'autre sexe. Le sexe féminin ayant un caractère de vide, comporte une part réelle, non symbolisable qui échappe à la fonction phallique. 
Cette énigme, cette part innommable du féminin, sur laquelle Lacan met l'accent et que Freud (1932) lui-même a fini par qualifier de "continent noir", a toujours suscité des questions depuis le début indatable de la réflexion humaine. Elle n'a cessé d'être suppléé par des fictions. Les rites et les mythes collectifs, tout comme ceux, individuels, du névrosé, ne sont d'ailleurs rien d'autre que des interrogations sur ce qu'est une femme, un homme, des interrogations sur le sexe, sur la mort...

Or qu'en est-il de cette énigme face aux mutations culturelles, lorsque les repères qui permettent au sujet de donner du sens au féminin et au masculin se transforment? La pratique clinique auprès de sujets immigrés, confrontés à un monde nouveau, qui n'abrite plus les mêmes rites et les mêmes ancrages symboliques, est riche en enseignements. Je chercherai à démontrer, en prenant appui sur ma pratique orientée par la psychanalyse et basée sur l'écoute du sujet dans son articulation au lien social, que ces transformations de repères ne sont pas sans répercussions subjectives. Elles entrent en résonance avec les remaniements psychiques. L'expérience montre en effet que l'énigme du féminin et du rapport à l'Autre sexe se réactualise avec acuité dans les moments de passage (grandir, devenir femme, mère, etc.) qui rappellent à chacun son incomplétude et sa condition d'être sexué. Cette épreuve peut concerner tout sujet, quelle que soit son appartenance culturelle, mais elle se redouble dans le contexte de l'immigration et peut exposer à des risques de défaillance lorsqu'aucun étayage symbolique ne vient border l'angoisse qu'elle génère.

Cette angoisse nécessite pour être apaisée un appel au mythe, à une fiction, afin de se préserver contre l'insoutenable du réel. Les voies d'accès au désir et à la parole peuvent ainsi s'ouvrir de nouveau. Mais les rites et les fictions qui en découlent sont parfois abandonnés en terre d'accueil ou alors reproduits mais non transmis, c'est-à-dire qu'ils se réduisent à des usages déconnectés de leur valeur symbolique. Ils ne sont pas accompagnés de récits et de mise en sens. C'est ce qui m’a été donné d'entendre chez certains sujets immigrés dont la souffrance, comme nous le verrons, met en jeu le rapport problématique à l'identité sexuée et à l'énigme du féminin.

Pour mieux comprendre cette problématique, je propose de prendre en compte dans un premier temps ce qui sous-tend la fonction des rites et les ressources mises en œuvre dans certaines cultures (Afrique, Maghreb) pour gérer le rapport à l'énigme du sexe et accompagner collectivement chaque sujet dans son devenir féminin et masculin. Dans un second temps, nous nous intéresserons au devenir de ces rites en terre étrangère ainsi qu'aux incidences cliniques qui leur sont liées.

Psic. Clin., Rio de Janeiro, vol. 25, N.I, P. I 97 - 2 I I, 2013 


\section{La fonction du rite face à l'énigme du féminin et du sexuel}

La plupart des travaux consacrés à la dimension du rite (anthropologique, sociologique ou psychologique), à commencer par ceux de Lévi-Strauss (1958/1995), sont d'accord pour dire que le rite ouvre le chemin de la symbolisation. Toutes les cultures construisent des montages afin d'apaiser l'angoisse et de se tenir à distance de l'insoutenable du réel. Qu'il soit religieux ou laïque, traditionnel ou modernisé, le rite répond à une nécessité: protéger, humaniser, constituer un rempart face à l'insaisissable qui habite irréductiblement tout sujet. Cet insaisissable, c'est ce qui est célébré à travers des nominations, des images et des symboles depuis que l'homme parle; ainsi se tisse et se reconstruit le lien social qui n'est autre qu'un lien langagier. Ce lien repose sur un interdit fondamental: l'interdit de l'inceste, impliquant la non-confusion des sexes et des générations. L'homme ne peut pas vivre sans ces remparts qui le lient à l'autre et qui lui fournissent des appuis identificatoires.

Toutefois, le rite échoue à désigner la chose qu'il convoque et qu'il prétend désigner. Ce qu'il désigne est toujours subverti, car pris dans une forme, une image, avec l'incessante revendication d'une identité. Il est donc toujours à reconstruire, car il n'est pas à l'abri de dérapage quant à son but. Nous pouvons dire que le rite a un aspect biface: dévoiler une réalité Autre, que M. Eliade (1976/1992) dénomme hiérophanie, soit étymologiquement ce qui est monstration, hors notre appartenance au monde, et la voiler par code et convention de langage. Parmi les montages culturels destinés à faire face à l'énigme du sexe, il y a la circoncision et le rite de nubilité. Dans le monde hébraïque, la circoncision est signe d'alliance. À la perte du prépuce - qui constitue le symbole féminin chez le garçon - au huitième jour de la naissance de l'enfant, se substitue le don du nom. Mais, comme dans le monde musulman, la circoncision permet de renouveler sans le clôturer le sens de la question: qu'est-ce qu'un homme, qu'est-ce qu'une femme? Il s'agit d'une marque (la circoncision en tant que coupure du prépuce) dans la chair de tous les mâles. Marque qui est avant tout à entendre comme l'acte par lequel la différence sexuelle doit être située du côté du manque, de la coupure.

\section{Le féminin et la marque du cycle du sang}

La circoncision marque un temps fort dans la vie d'un garçon. Pour la jeune fille, c'est le surgissement des premières règles qui vient faire marque dans son corps, ce qui n'est pas sans angoisse. Cette question ne passse pas inaperçue pour Freud 
qui, dès les premiers balbutiements de la psychanalyse, écrira à ce propos dans une lettre à Fliess: "C'est maintenant seulement que je commence à comprendre la névrose d'angoisse" (Freud, 1896-1902/1973, p. 141). Avec l'apparition des règles s'ouvre en effet pour la jeune fille une nouvelle temporalité obéissant à une loi de la répétition rythmée par le cycle menstruel. À ce temps spécifique, à cette période de nubilité où la fillette, devenant femme, rencontre le réel de son organisme, une initiation est parfois consacrée. Ainsi, dans certaines régions du Maghreb, le corps de la jeune fille devient à ce moment-là surface d'inscription. Le henné ou parfois le tatouage (wachma), désigné aussi par l'expression "écriture en point", viennent rendre compte d'une mutation, d'une nouvelle position du féminin.

Il est important de noter que l'Islam interdit le tatouage. Or, cette pratique millénaire est non seulement maintenue, mais elle s'effectue paradoxalement au nom de Dieu, ce qui est une manière, selon A. Khatibi (1974, p. 102), de "substituer à l'ordre de Dieu la stratégie humaine du désir". La différence entre le henné et le tatouage, c'est que l'un, provisoire, est dessiné sur l'épiderme avec un bout de bois fin, et l'autre, durable, est tracé sur le corps avec une aiguille. Pourtant, l'un comme l'autre sont destinés à protéger contre l'insoutenable du réel et à inscrire sur le corps ce qui détermine l'être féminin. Ces inscriptions corporelles ont également lieu au moment du mariage. Ces opérations à travers lesquelles se met en scène la séparation des sexes et que l'on retrouve dans d'autres contextes culturels sont par ailleurs censées rendre possibles et pensables la sexualité et la procréation.

Chez les Nuers d'Afrique Orientale (éleveurs du Soudan), le féminin est inconcevable en dehors du maternel. La "stérilité", vécue comme l'une des choses les plus graves et qui porte atteinte au lien social, doit modifier le statut d'une personne, indépendamment du sexe anatomique, afin de rétablir un ordre, une régulation, un équilibre. Dans cette société, les femmes infertiles sont considérées comme des hommes. Elles peuvent prendre des épouses qu'un homme de la tribu peut féconder, mais ce seront elles qui seront appelées père par leur enfant ou mari par leur femme. Les rites, les cultes, voire les idéologies, quelles que soient leurs formes et leurs cérémonies convenues, donnent ainsi du sens à l'impensable. Mais ils peuvent aussi basculer vers la violence et la barbarie lorsque leur usage supplante leur fonction de nouage, leur dimension symbolique.

\section{Le devenir du rite en terre étrangère}

La question qui se pose ici c'est: que deviennent ces rites, dans lesquels chaque sujet s'implique singulièrement, face aux changements culturels, pour

Psic. Clin., Rio de Janeiro, vol. 25, N.I, P. I 97 - 2 I I, 2013 
ceux qui se retrouvent dans un contexte étranger pour avoir choisi d'habiter loin de la terre natale ou parce qu'ils ont été contraints à l'émigration? Comment peuvent-il assurer leur fonction protectrice dans un contexte non soutenu par un étayage familier, qui vient bousculer le sujet en exil dans son rapport à sa culture, à ses fictions, dans ce qu'elles lui offrent comme moyens pour pallier au manque à être et réguler son rapport au désir, à la différence des sexes et des générations?

Ainsi que je l'ai montré par ailleurs (Sitou, 1997, p. 16), cette épreuve de l'extranéité réactualise inévitablement le "qui suis-je?" au niveau de l'identité, question qui ne peut trouver de réponse qu'à travers un récit qui aide chacun à trouver des repères dans ses références mythiques. A travers cette épreuve, se repose avec acuité la question de l'origine, de l'appartenance et de la transmission qui exige des réaménagements permettant l'investissement d'autres codes et le tissage de nouveaux liens. Si l'expatriation peut conduire certains à trouver la voie de la sublimation, pour d'autres le vécu dramatisé de cette expérience les confronte à une perte de sens et au vacillement des repères. En effet, le changement de pays, de langue et de culture peut raviver, parfois avec violence, les failles subjectives et les blessures infantiles. Mais comment tenir à distance une telle violence lorsque le sujet se sent exclu de l'univers porteur de ses croyances ou au contraire lorsqu'il s'y agrippe d'une manière extrême?

L'épreuve de l'inconnu actualisée par l'expatriation et à travers laquelle se produit parfois une mise à nu du sujet, peut avoir une portée dramatique lorsque rien ne vient border l'angoisse qu'elle engendre. Les difficultés que connaissent les familles expatriées que j'ai rencontrées (essentiellement maghrébines ou africaines) se redoublent souvent en se faisant plus intenses à chaque épreuve de l'existence (grandir, devenir femme ou homme, accéder à la maternité), c'est-à-dire dans ces temps de passage et de remaniements psychiques qui confrontent à l'incomplétude ou au manque à être, mais sans parvenir à être symbolisés. C'est comme si les constructions fantasmatiques, les références signifiantes de ces sujets étaient en faillite dans un contexte qui n'abrite plus les mêmes fictions. Précisons que dans les sociétés dites traditionnelles, la puberté et le devenir féminin sont considérés comme une période d'incertitude qui expose à tous les dangers, d'où la nécessité du rite. En grandissant, l'enfant doit changer de place mais oblige également ses parents à se déplacer et à se rendre au temps comme êtres parlants, inscrits dans une filiation, mortels et sexués. Il s'agit là d'une véritable mutation qui nécessite de passer par la déconstruction/reconstruction des représentations permettant habiter autrement son corps, permettant s'inscrire dans un nouveau rapport à soi et à l'autre.

Le rite autour duquel se mobilise toute la collectivité autorise l'enfant grandissant à traverser un temps d'errance et d'indécision quant à son appartenance, 
impliquant une nouvelle identification sexuée ainsi qu'une ré-appropriation du lien aux autres. Après ce temps d'errance reconnu par la société qui permet par là-même au sujet de se reconnaitre à la fois différent et semblable aux autres, intervient le temps d'agrégation (Van Gennep, 1909) qui constitue une sorte de reconsécration identitaire. Parents et proches sont amenés à déplacer leur regard sur l'adulte et la femme en devenir, acceptant son énigme, en lui donnant une autre place, à partir de nouvelles limites qui inscrivent un nouvel ordre d'échanges. Ce déplacement du regard est associé à des paroles porteuses de repères symboliques. C'est ainsi que le rite tient à distance le chaos et la pulsion de mort.

L'expérience clinique auprès de sujets immigrés montre que lorsque ces rites se réduisent à de simples usages désarrimés de toute mise en sens, ils perdent leur efficacité symbolique. Cela n'est pas sans incidences subjectives, surtout lorsque le sujet ne se réfère pas à d'autres repères ou modèles identificatoires lui permettant de se reconnaître dans sa condition d'être sexué et d'accepter la différence. Les rites et les croyances qui entourent ces moments-là sont, pour certains sujets, abandonnés une fois en terre d'accueil ou exhibés en tant que signes folkloriques. Il y a ceux qui rejettent leurs croyances avec leur différence pour s'adapter au pays d'accueil: ils ne conçoivent que de la honte et de la culpabilité de ne pas être conformes aux autochtones, ce qui témoigne d'un sentiment d'identité en faillite. Cette assimilation forcée se répercute souvent sur les enfants qui sont confrontés à une impasse au niveau de la transmission. La clinique tend à nous montrer que ces enfants, dont chaque cas est à entendre bien sûr au singulier, ne reçoivent de leurs références culturelles et langagières que le silence. Un silence parfois mortifère qui ne leur permet pas de s'approprier leur histoire, de l'élaborer afin de s'ouvrir à d'autres liens. Ils sont exclus de tout récit concernant l'ailleurs dont ils sont porteurs. Leur souffrance m'a souvent été donnée à entendre à travers l'inhibition à construire, à s'inscrire dans le rapport à l'Autre, y compris l'autre sexe.

Il y a également des parents qui vont se raccrocher d'une manière défensive, voire caricaturale, à leurs traditions en devenant plus africains que les Africains, plus musulmans que les Musulmans. Ils se figent dans le donné à voir de leurs traditions, mais en les désarrimant de toute fiction. Ainsi les rites de passage, qui sont censés permettre à l'enfant grandissant de métaphoriser les coupures qu'il traverse, se réduisent en terre étrangère à des signes clivés de tout lien langagier. L'enfant musulman va par exemple être circoncis, mais l'acte rituel, destiné à rendre signifiante cette marque dans la chair, capable de présentifier du côté du manque la différence des sexes et des générations, se transforme en agir. Il devient un agir médicalisé qui ne porte aucun sens. Autrement dit, l'usage qui consiste

Psic. Clin., Rio de Janeiro, vol. 25, N.I, P. I 97 - 2 I I, 2013 
à couper le prépuce supplante l'étayage symbolique sans lequel aucune possibilité de déplacement métaphorique n’est offerte. Dans ce cas-là, la circoncision peut être vécue par l'enfant non pas comme quelque chose de structurant mais comme une mutilation, une violence incommensurable qui l'empêche d'investir son corps comme un corps sexué et désirant.

Cet exemple du devenir de la circoncision en terre étrangère est valable pour tous les rites, dont le rite de nubilité concernant le devenir féminin. Ces rites sont délaissés et/ou ne s'inscrivent plus dans une logique de transmission mais de reproduction, c'est-à-dire qu'ils ne sont plus accompagnés par des médiations, des sens et des nominations, ce qui peut entrainer quelques incidences subjectives. La période de nubilité peut en effet dans ce cas se transformer en désordre. Au lieu de susciter quelque chose de constructif, elle devient source de mal-être et de souffrance, aussi bien pour la fillette devenant femme que pour ses parents. Certains parents expatriés vivent l'énigme du féminin qu'incarne leur fille comme une nouvelle "épreuve de l'étranger" (Berman, 1984).

L'épreuve de l'étranger les renvoie non seulement à l'immigration et à la différence culturelle, mais aussi à cette épreuve de l'innommable inscrite au cœur du sujet, inhérente à l'Autre scène qu'est l'inconscient. Cette épreuve n'est pas sans risque de violence lorsqu'aucune médiation ne vient métaphoriser ce passage du devenir féminin en conjuguant la dialectique de ce qui relève de la subjectivité et de la réalité culturelle, du proche et du lointain, du familier et de l'étranger. Face à l'absence ou à la fragilité de cette médiation symbolique, les parents immigrés reportent, voire confondent l'étrangeté liée au féminin et à la part obscure du sujet avec l'autre non semblable, le différent culturellement. L'étrangeté se trouve alors vécue dans sa version insupportable. La fillette qui grandit et dont le corps se métamorphose leur échappe. Elle devient étrangère, ce qu'ils acceptent très difficilement. Plusieurs parents nous demandent d'ailleurs d'aider leur fille à redevenir comme avant, à revenir à l'image de l'enfant merveilleux, inchangé et inchangeable. Cela ne va pas sans angoisse et c'est souvent à cette période qu'ils imposent à leur progéniture le retour au pays d'origine, comme si avec ce retour à la culture "d'origine", supposée immuable, ils allaient se débarrasser de l'inconnu et retrouver du même, de l'identique. C'est l'imaginaire qui prend ici le dessus. Cette façon de s'agripper non pas à la fonction symbolique de la culture mais à ce qu'ils imaginent être non modifiable, déstabilise les enfants dans leurs repères identificatoires.

Je pense ici à Lina, adolescente d'origine turque âgée de quinze ans. Je l'ai rencontrée une seule fois au CMPP (Centre médico-psycho-pédagogique) ${ }^{2}$, après avoir reçu ses parents, le temps d'une écoute et d'un regard qui reconnaît 
l'étrangeté en elle et hors d'elle, non pas comme une menace à congédier mais comme ce qui la renvoie à ce qu' elle a de plus intime. Lina a en effet été rencontrée le temps de faire le point sur ses difficultés, à la demande de son médecin. Elle a ensuite été orientée vers un lieu plus proche de son domicile, dans une localité voisine, pour un travail psychothérapeutique.

\section{Fragment clinique}

Devenue méconnaissable aux dires de ses parents, elle aurait déstabilisé toute la famille par ses conduites étrangères aux mœurs et aux lois de leur pays (sorties fréquentes, extravagances...). Les éléments culturels sont d'emblée mis de l'avant pour rendre compte de l'étrangeté dont leur fille est porteuse et de l'immense souffrance que cela génère. Il s'agit là d'un rapport défensif à la culture auquel il est important d'être attentif car, à travers lui, se donne à entendre l'implication de chacun et ce qu'il y a de symptomatique dans le lien familial et de transmission. La non-conformité de leur fille à ce que leurs traditions déterminent comme "être femme", c'est ce que les parents présentent donc comme étant leurs principales préoccupations. Et pourtant Lina dira la même chose de ses parents en insistant sur ce qu'elle éprouve comme un sentiment d'étrangeté. Elle ne les comprend pas et dit se sentir étrangère à la culture turque dont elle ne sait rien en dehors des plats que cuisine sa mère ou encore des contraintes et des injonctions blessantes.

La culture turque transmise par sa mère a donc été investie comme une blessure. Elle dira plus tard cependant, lorsqu'elle parviendra à renouer avec la parole, avoir été marquée durant sa petite enfance par les contes et histoires turques racontés par sa grand-mère paternelle, les rares fois où celle-ci a quitté Istanbul pour venir les voir. Cela m’apaisait et me faisait rêver”. Mais étrangère, elle l'est aussi à elle-même, car elle dit ne pas se reconnaître dans ses moments d'angoisse. Lina vit très difficilement sa féminité, et ses parents aussi. Ils la regardent comme si elle était porteuse d'un insupportable. Et c'est ce regard qui la perturbe dont elle tente de se défaire. C'est donc autour de cette insupportable féminité, reportée sur la non-conformité à la culture du pays d'origine, qu'émerge la souffrance de Lina et que se cristallisent les conflits avec ses parents. L'arrivée de ses premières règles est décrite comme un événement traumatique. Le rite destiné à entourer ce moment de passage où se manifeste le réel du corps, en le nouant à l'imaginaire et au symbolique, n'a pas été présentifié par sa famille, et plus particulièrement par sa mère qui pourtant ne cesse de mettre

Psic. Clin., Rio de Janeiro, vol. 25, N.I, P. I97 - 2I I, 2013 
en avant ses traditions. Cette dernière exprimera d'ailleurs son regret de ne pas avoir accompli de cérémonie rituelle et précisera que l'absence de la famille élargie l'avait découragée. Lina était donc en panne d'un récit lui permettant de tenir à distance cet événement du corps et de symboliser ce qui fait manque dans la fiction.

Rappelons que le rite de nubilité permet non seulement de donner du sens à l'énigme du féminin et du sexuel, mais il assure une fonction de reconsécration identitaire. Ce rite initiatique est donc consacré à la fois à la jeune fille et à son entourage qui la regarde autrement car grandir, devenir femme, n’est pas une affaire privée mais un événement à partager, inscriptible dans le lien social, qui permet à la famille de se rassembler autour de ce qui demeure énigmatique et impensé afin de le métaphoriser. Or qu'en est-il lorsque cette énigme devient insoutenable? Non seulement le rite n'a pas eu lieu pour Lina, mais il a cédé place à des propos d'une violence ravageante de la part de sa mère: "Tu es devenue une femme, il va falloir te surveiller de très près". Depuis, rien ne va plus dans son rapport à l'Autre parental et à son corps, qui ne se manifeste qu'à travers la douleur abdominale, les maux de tête, la tension nerveuse. Face à l'inefficacité des antalgiques, le médecin pense prescrire un contraceptif. Selon lui, Lina souffre d'une forme sévère de syndrome prémenstruel. Il lui conseille également de voir un psychiatre, car cela peut masquer une dépression. La mère de Lina refuse le contraceptif qui peut exposer sa fille à l'égarement, à une dérive sexuelle. Pour elle, la seule issue est le retour au pays d'origine.

Craignant que la menace de ses parents (retour dans le pays d'origine) ne soit mise à exécution, Lina a demandé l'aide d'une assistante sociale. Affectée par cette demande, l'assistante sociale a entrepris les démarches nécessaires pour un placement en foyer. Mais Lina s'est rétractée, ne supportant pas l'idée de voir sa famille malheureuse. Les parents, contactés par le juge, se sont effondrés. C'est dans cette atmosphère de drame que Lina a tenté de mettre fin à ses jours. C'est dans l'agir, un agir symétrique à celui de la famille et de l'assistante sociale, que Lina tente d'échapper au tiraillement entre le vœu de maintenir les liens avec ses parents et la tentative d'affirmer sa singularité.

L'incertitude propre à l'adolescence, que vient ici redoubler l'énigme du féminin, provoque une angoisse qui crée la nécessité de passer par des repères symboliques, mais qui ne rencontre en guise de réponse qu'un discours objectivant qui la met de côté en tant que sujet. La tentative de suicide peut être entendue comme une tentative de s'expulser du lieu que les autres lui imposent, ou dans lequel ils tentent de la ramener, sans que pour autant elle trouve sa place. Il s'agit d'un appel, d'une monstration de ce qui ne peut se dire. Son mal-être, 
l'expression de la douleur qui accompagne le surgissement des règles, n'a pu être entendable en dehors de la dimension organique ou biologique. Face à la butée de la rationalité médicale, les parents, et plus particulièrement la mère, ont mis en avant le recours aux traditions, un recours désarrimé de toute dimension symbolique, et qui au contraire fait violence à Lina.

D'un côté ses parents exigent le retour au même (à travers le retour aux origines, la Turquie) face à l'insupportable étrangeté que leur renvoie la fille devenant femme. C'est le modèle occidental qui, selon eux, les dépossède de leur enfant. D'un autre côté, en répondant au patent de la demande de Lina, et pensant bien faire, l'assistante sociale désapprouve ce que les parents de l'adolescente présentent comme leurs repères culturels et lui propose un autre lieu d'assimilation factice. Des deux côtés, Lina est assignée à une place qui n'est pas la sienne, mais celle d'un fantasme de complétude ou d'un conformisme qui l'empêche d'occuper une position de sujet à partir de ce qui la divise et fait mystère pour elle.

Or, ce que réclame Lina, c'est d'être reconnue dans sa découverte singulière du féminin, afin de se reconnaître elle-même, d'affronter l'inconnu et de s'ouvrir à l'altérité. Comment se reconnaître si l'autre perçoit en elle une menace ou ne l'accepte pas avec ce savoir énigmatique dont elle est porteuse? Précisons que c'est le regard des parents, mais aussi social, qui permet à l'enfant (et cela se rejoue à la puberté, au moment de la nubilité et de l'adolescence), de se reconnaître, de fabriquer ses identifications en intégrant la frontière qui le lie et le sépare de l'autre. Aucun sujet ne peut se reconnaître si l'Autre ne le reconnaît pas. Mais lorsque le regard de l'Autre devient harceleur ou persécuteur, lorsqu'il n'engage pas le désir, il peut conduire à la violence car, paradoxalement, cette dernière émerge lorsque le sujet se sent menacé dans ce qui le protège et l'humanise.

Aucune parole consistante ne semble soutenir Lina dans ce temps de passage adolescent qui, en ravivant les blessures infantiles, en bouleversant le rapport au corps, en bousculant les montages imaginaires autour du mystère du sexe, de l'origine et de la mort, nécessite un travail d'élaboration psychique. En n'acceptant pas le changement de Lina, l'entourage lui impose au contraire de rester sédentaire dans ses difficultés. "Il fallait que ça déménage" dira d'ailleurs Lina. Cette non-reconnaissance peut être ravageante. "Je n'ai pas peur de mourir" dira encore Lina. "Ce qui me tue, c'est le regard de mes parents". Ce regard de l'Autre, nécessaire à la reconstruction des identifications, s'avère problématique pour Lina. À travers lui, elle se sent dénigrée en tant que femme en devenir. Tantôt fuyante, tantôt s'agrippant à mon regard comme pour vérifier sa consistance à mes yeux, Lina exprimera tout au long de notre rencontre son sentiment

Psic. Clin., Rio de Janeiro, vol. 25, N.I, P. I 97 - 2 I I, 2013 
d'être désorientée: "Si j'étais un garçon ou une Française, je n’aurais pas vécu ce calvaire".

C'est autour d'un bracelet que je portais ce jour-là que s'est nouée la relation. Attirée par cet objet dont les motifs "orientaux" l'ont renvoyée à quelque chose "d'extime" (ce qui évoque à la fois l'étranger et le familier), Lina a pu ouvrir la question de ses origines, de son histoire et de sa position féminine. Le surgissement de cette brèche a été l'occasion de lui indiquer un lieu de mise au travail, qui lui donne la possibilité de nommer ses blessures, c'est-à-dire de les transformer en plainte puis en message adressé à l'Autre dans le transfert. C'est ainsi qu'elle peut réinvestir son corps comme vivant, désirant, sexué.

Ce fragment clinique nous montre que la construction de la féminité constitue une épreuve à la croisée du singulier et du collectif, qui se complexifie parfois dans le contexte de l'immigration au point où l'étrangeté à laquelle confronte cette dimension énigmatique du féminin se redouble face à l'étrangeté et à la différence culturelle. Et c'est souvent à travers la mise en avant de la culture que le sujet donne à entendre sa souffrance et l'épreuve singulière qu'il traverse.

Cette épreuve peut être structurante lorsqu'elle parvient à se métaphoriser et à ouvrir sur l'altérité. Mais elle peut aussi exposer à des risques de défaillance lorsqu'elle est vécue comme quelque chose de dramatique, non étayé par une parole constructive. Le rapport au féminin et à la différence des sexes est lié à la subjectivité, mais il porte aussi la marque d'une culture dont les formes expressives ne cessent de changer en fonction des temps et des lieux. L'expérience clinique peut révéler que la manifestation du mal-être du sujet expatrié n'est pas la seule à questionner sa connexion avec la dimension socioculturelle. L'expression de la souffrance du sujet autochtone, occidental, sujet de la modernité par excellence, génère elle aussi des questions face aux changements culturels, aux mutations des idéaux et des modèles identificatoires qui permettent à chacun de s'y retrouver, de se reconnaître dans sa condition d'être sexué et d'accepter la différence.

Le sujet immigré ne fait que révéler, à travers l'écart qu'introduit sa différence, cette nécessité de prendre en compte l'articulation entre la dimension subjective et culturelle. Les références culturelles ne sont donc pas à négliger dans notre écoute clinique, mais à condition de ne pas confondre la culture, qui n'existe pas indépendamment du sujet, et le culturalisme qui consiste à recouvrir l'inconnu d'images exotiques. Autrement dit, ce sont moins les usages des rites, des mythes et des croyances qui importent que la manière dont chaque sujet s'y implique singulièrement, avec son histoire, pour fabriquer ses identifications, se lier à l'autre et se soutenir dans le monde. 


\section{Références}

Assoun, P. L. (1993). Freud et les sciences sociales. Paris: Armand Colin.

Blanchot, M. (1983). La communauté inavouable. Paris: Editions de Minuit.

Berman, A. (1984). L'épreuve de l'étranger. Paris: Gallimard.

Eliade, M. (1988). Initiations, rites, sociétés secrètes. Paris: Gallimard. (Publié originalement en 1976)

Freud, S. (1969). L'organisation sexuelle infantile. In La vie sexuelle. Paris: PUF. (Publié originalement en 1923)

Freud, S. (1973). Naissance de la psychanalyse. Paris: PUF. (Publié originalement en 1896-1902)

Freud, S. (1989). XXXIIIe conférence: La féminité. In Nouvelles conférences d'introductions à la psychanalyse. Paris: Gallimard. (Publié originalement en 1932)

Freud, S. (1993). L'avenir d'une illusion. Paris, PUF. (Publié originalement en 1928)

Gori, R. \& Del Volgo, M. J. (2008). Exilés de l'intime: la médecine et la psychiatrie au service du nouvel ordre économique. Paris: Denoël.

Khatibi, A. (1974). La blessure du nom propre. Paris: Denoël.

Lacan, J. (1975). Le séminaire Livre XX. Paris: Seuil. (Séminaire de 1972-1973)

Legendre, P. (1992). L'inestimable objet de la transmission. Paris: Fayard.

Lévi-Strauss, C. (1995). Anthropologie structurale. Paris: Plon. (Publié originalement en 1958)

Nathan-Murat, M. (1991). Anorexie mentale et excision: leur rapport au renoncement à la mère phallique. Revue du collège des psychanalystes, 41, 89-96.

Rassial, J. J. (1996). Le passage adolescent: de la famille au lien social. Ramonville-St-Agne: Erès. Stitou, R. (1997). Universalité et singularité de l'exil. Psychologie clinique, 3, 13-29.

Thomas, L.V., Lumeau, R. (1986). La terre africaine et ses religions. Paris: L'Harmattan.

Van Gennep, A. (1909). Les rites de passage. Paris: Nourry.

Winnicott, D. W. (1989). L'enfant et le monde extérieur, le développement des relations. Paris: Payot. (Original publicado em 1957)

\section{Notes}

${ }^{1}$ Le désir ne peut déboucher sur une totale satisfaction. Le sujet fait non seulement l'expérience de son incomplétude, c'est-à-dire du manque au cœur de son être, mais aussi de l'impossible complémentarité entre les sexes. Contrairement au règne animal, la rencontre d'un homme et d'une femme n'a pas la simplicité d'une rencontre entre un mâle et une femelle.

${ }^{2}$ Le Centre Médico-Psycho-Pédagogique assure une fonction d'accueil, d'écoute et de soins sous forme ambulatoire auprès d'enfants et d'adolescents présentant des difficultés psychoaffectives, scolaires et relationnelles. Sa mission est de prendre en compte la souffrance de

Psic. Clin., Rio de JANeiro, vol. 25, N.I, P. I 97 - 21 I , 2013 
l'enfant et de faciliter les relations avec son environnement familial, scolaire et social. Le CMPP peut également être consulté pour un simple avis pour un enfant ou un adolescent en situation ponctuellement préoccupante, ce qui a été le cas de Lina.

Recebido em 23 de julho de 2012 Aceito para publicação em 17 de novembro de 2012 